\title{
Myrsinaceae endémicas del Perú
}

Blanca León ${ }^{1,2}$

${ }^{1}$ Museo de Historia Natural,
Av. Arenales 1256, Aptdo.
14-0434, Lima 14, Perú
${ }^{2}$ Plant Resources Center,
University of Texas at
Austin, Austin TX 78712
EE.UU.
blanca.leon@mail.utexas.edu

\section{Resumen}

La familia Myrsinaceae es reconocida en el Perú por presentar nueve géneros y 92 especies (Brako \& Zarucchi, 1993; Ulloa Ulloa et al., 2004), la mayoría arbustos y árboles. En este trabajo reconocemos 28 especies endémicas, en cinco géneros. Las especies endémicas se encuentran principalmente en Bosques Húmedos Amazónicos y Bosques Muy Húmedos Montanos, entre los 100 y 3400 m de altitud. Diez especies endémicas se encuentran dentro del Sistema Nacional de Áreas Naturales Protegidas por el Estado.

Palabras claves: Myrsinaceae, Perú, endemismo, plantas endémicas.

\section{Abstract}

The Myrsinaceae are represented in Peru by nine genera and 92 species (Brako \& Zarucchi, 1993; Ulloa Ulloa et al., 2004), mostly shrubs and trees. Here we recognize 28 endemic species in five genera. Endemic species are found mainly in Humid Lowland Amazonian Forests and Very Humid Montane Forests regions between 100 and $3400 \mathrm{~m}$ elevation. Ten endemic species of Myrsinaceae have been registered to date within Peru's protected areas system.

Keywords: Myrsinaceae, Peru, endemism, endemic plants.

\section{Ardisia martinensis Lundell}

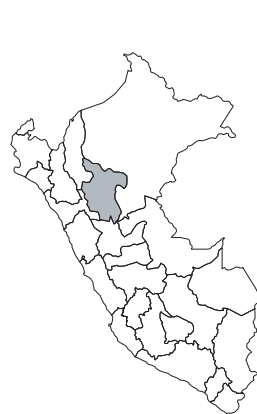
$\mathrm{m}$.

\section{CR, B1ab(iii)}

Publicación: Wrightia 6(4): 82. 1979. Colección tipo: J. Schunke V. 7670

Herbarios: TEX-LL.

Nombre común: Desconocido.

Registro departamental: SM.

Regiones Ecológicas: BMHP; 500—850

SINANPE: Sin registro.

Herbarios peruanos: Ninguno.

Observaciones: Árbol conocido, al parecer, sólo de la colección tipo, una planta proveniente de la cuenca del Huallaga y recolectada en 1974. La parte media de esa cuenca, en la que se ubica la localidad tipo, ha sido extensamente modificada por actividades agrícolas.

\section{Ardisia nigrovirens J.F. Macbr.}

\section{DD}

Publicación: Candollea 5: 397. 1934.

Colección tipo: L. Williams 5081

Herbarios: F.

Nombre común: Desconocido.

Registro departamental: LO, MD.

Regiones Ecológicas: BHA; 155-400 m. SINANPE: PNM

Herbarios peruanos: Ninguno.

Observaciones: Esta especie arbustiva fue descrita de una planta recolectada en la cuenca del bajo Huallaga, en 1929. Ha sido recolectada en otras localidades, en forma disyunta, en el sur de la Amazonía peruana y en el Parque Nacional Manu.

\section{Cybianthus candamoensis Pipoly \& Ricketson}

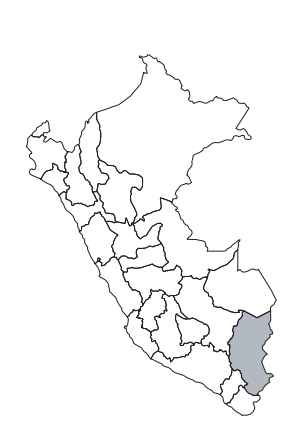

\section{CR, B1a}

Publicación: Novon 16(2): 251-253, f. 2. 2006.

Colección tipo: F. Cornejo \& A. Balarezo 2947

Herbarios: MO; MOLF.

Nombre común: Desconocido.

Registro departamental: PU.

Regiones Ecológicas: BHA; $400 \mathrm{~m}$.

SINANPE: RNT

Herbarios peruanos: MOLF (isotipo citado).

Observaciones: Arbusto dioico, conocido y descrito solamente de una planta masculina. Esta especie es conocida de la cuenca del Candamo en la Reserva Nacional Tambopata. Por lo limitado de su distribución y población reducida, Pipoly \& Ricketson (2006) la consideraron en Peligro Crítico, conclusión con la que se concuerda.

\section{Cybianthus flavovirens Pipoly}

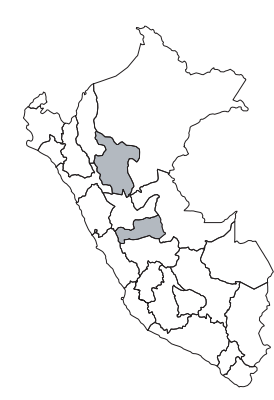

\section{EN, B1ab(iii)}

Publicación: Sida 18(1): 134, 136. 1998. Colección tipo: J. Schunke V. 10883 Herbarios: BRIT, F, MO, TEX, US; AMAZ, USM.

Nombre común: Desconocido.

Registro departamental: PA, SM.

Regiones Ecológicas: BMHP, BHA; 300-800 m.

SINANPE: Sin registro.

Herbarios peruanos: AMAZ (isotipo citado), USM (isotipo citado).

Observaciones: Árbol bajo, conocido, al parecer, de dos localidades. El ejemplar tipo proviene de la cuenca del Huallaga medio. Otro ejemplar fue recolectado de los bosques bajo manejo forestal, en la década de los 1980, en la cuenca del Palcazú. En ambas localidades, la deforestación es una amenaza a sus poblaciones. Pipoly (1998) sugirió clasificar esta especie como «En Peligro» por la distribución restringida y las presiones a su hábitat, criterio con el concordamos. 


\section{Cybianthus fosteri Pipoly}

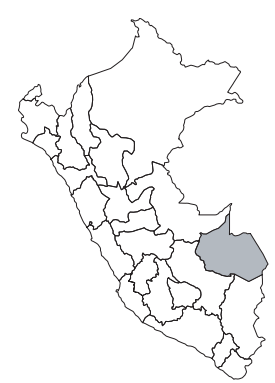

EN, B1a

Publicación: Sida 18(1): 144. 1998.

Colección tipo: R.B. Foster \& T. Wachter 7254

Herbarios: F, NY; USM.

Nombre común: Desconocido.

Registro departamental: MD.

Regiones Ecológicas: BMHP; 600-900 m.

SINANPE: Sin registro.

Herbarios peruanos: USM (isotipo).

Observaciones: Árbol conocido, al parecer, sólo de la localidad tipo, ubicada al sur del Parque Nacional Manu. El ejemplar tipo de esta especie fue recolectado en bosques de colina, en 1983; los cuales han sido escasamente estudiados, pero se hallan amenazados por la deforestación.

\section{Cybianthus grandezii Pipoly}

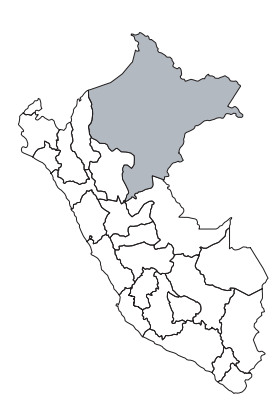

\section{DD}

Publicación: Sida 18(1): 139. 1998.

Colección tipo: C. Grández 1824

Herbarios: MO, US; AMAZ.

Nombre común: Desconocido.

Registro departamental: LO.

Regiones Ecológicas: BHA; $125 \mathrm{~m}$.

SINANPE: Sin registro.

Herbarios peruanos: AMAZ (isotipo citado).

Observaciones: Arbusto conocido, al parecer, sólo de la colección tipo, una planta recolectada en 1990, en la cuenca del Amazonas.

\section{Cybianthus granulosus Pipoly}

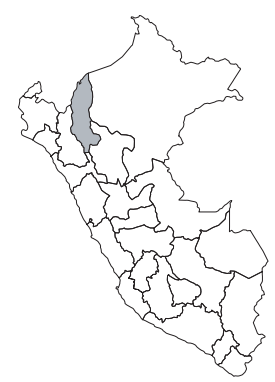

\section{DD}

Publicación: Sida 18(1): 133. 1998.

Colección tipo: S. Tunqui 161

Herbarios: NY.

Nombre común: Desconocido.

Registro departamental: AM.

Regiones Ecológicas: BHA; 180—200 m. SINANPE: ZRSC

Herbarios peruanos: Ninguno.

Observaciones: Arbusto, al parecer, restringido a la cuenca del Santiago, en la Zona Reservada Santiago-Comaina. Todos los registros conocidos fueron realizados en 1979 y poco se sabe de su hábitat.

\section{Cybianthus huampamiensis Pipoly}

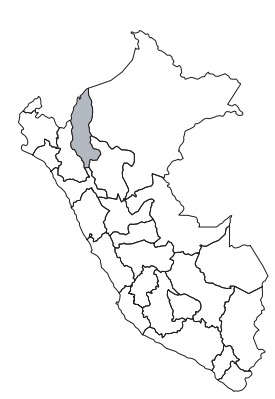

\section{NT}

Publicación: Sida 18(1): 130—133, f. 23. 1998.

Colección tipo: E. Ancuash A. 588

Herbarios: MO, NY; USM.

Nombre común: Desconocido.

Registro departamental: AM.

Regiones Ecológicas: BHA; 180-1950 m. SINANPE: ZRSC

Herbarios peruanos: USM (isotipo citado).

Observaciones: Árbol o arbusto, conocido de varias localidades, en las cuencas del Cenepa, Marañón y Santiago. Esta especie ha sido registrada en un área que, como señala Pipoly (1998), se halla en los planes de desarrollo, pero al mismo tiempo, ha sido muy poco botánicamente explorada.

\section{Cybianthus incognitus Pipoly}

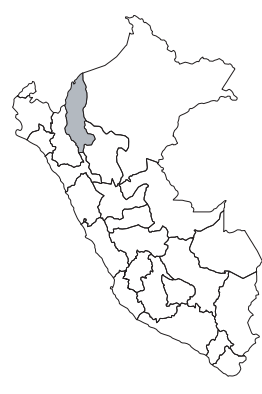

EN, B1a

Publicación: Sida 18(1): 125—127, f. 22. 1998.

Colección tipo: S. Tunqui 289

Herbarios: MO, NY; USM.

Nombre común: Desconocido.

Registro departamental: AM.

Regiones Ecológicas: BHA; 200-1700 m.

SINANPE: ZRCC, ZRSC

Herbarios peruanos: HUT (1), USM (isotipo citado).

Observaciones: Árbol conocido de las cuencas del Utcubamba y del Santiago. El ejemplar tipo proviene de la Zona Reservada Santiago-Comaina. En Cordillera de Colán, hoy Zona Reservada, esta especie tiene un rango altitudinal de casi $1500 \mathrm{~m}$; esta zona alberga varios endemismos y debería de ser de interés su conservación.

\section{Cybianthus jensonii Pipoly}

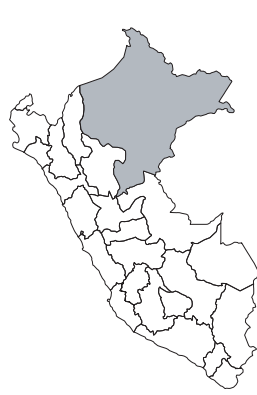

\section{EN, B1a}

Publicación: Sida 18(1): 142—144, f. 27. 1998. Colección tipo: A.H. Gentry 29700

Herbarios: MO, NY; AMAZ, USM!.

Nombre común: Desconocido.

Registro departamental: LO.

Regiones Ecológicas: BHA; $130-210 \mathrm{~m}$.

SINANPE: Sin registro.

Herbarios peruanos: AMAZ (isotipo citado+2), USM (isotipo).

Observaciones: Especie arbustiva conocida de unas pocas localidades en las cuencas del Amazonas, Napo y Pastaza. La localidad original está ubicada cerca al límite con Ecuador, por lo que podría estar representada en la flora de ese país. Pipoly (1998) sugiere que es una especie rara.

\section{Cybianthus nanayensis (J.F. Macbr.) G. Agostini}

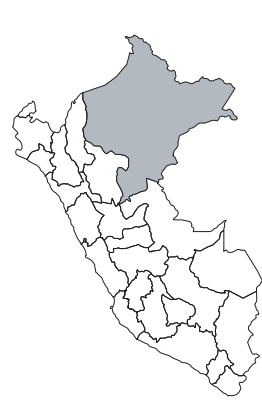

$$
\text { VU, B1ab(iii) }
$$

Publicación: Acta Biol. Venez. 10: 160. 1980. Colección tipo: L. Williams 658

Herbarios: F, G.

Nombre común: Desconocido.

Registro departamental: LO.

Regiones Ecológicas: BHA; 100—130 m. SINANPE: RNAM

Herbarios peruanos: Ninguno.

Observaciones: Arbusto conocido de localidades dispersas en los alrededores de Iquitos, como en las cuencas del Nanay y Napo. Habita bosques no inundables en diferentes tipos de suelos. Por lo menos dos de sus poblaciones son conocidas de áreas protegidas tanto del Estado (Reserva Nacional Allpahuayo-Mishana) como privadas (Explorama).

\section{Cybianthus nestorii Pipoly}

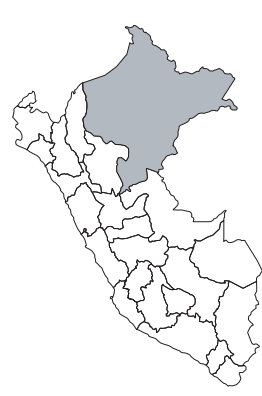

\section{CR, B1ab(iii)}

Publicación: Sida 18(1): 59—61, f.15. 1998. Colección tipo: R. Vásquez \& N. Jaramillo 6122

Herbarios: F, MO, NY, US; AMAZ, USM.

Nombre común: Desconocido.

Registro departamental: LO.

Regiones Ecológicas: BHA; $150 \mathrm{~m}$.

SINANPE: Sin registro.

Herbarios peruanos: AMAZ (isotipo), USM (isotipo citado). 
Observaciones: Árbol raro, conocido, al parecer, sólo de la localidad tipo, en la cuenca del río Nanay. Esta especie fue descrita de una planta recolectada en 1985 y vuelta a registrarse en 1993 de individuos marcados, en una parcela de inventario. Habita bosques no inundados sobre arena blanca.

\section{Cybianthus pseudolongifolius Pipoly}

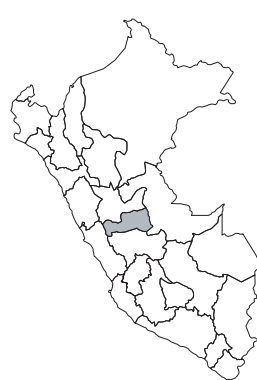

DD

Publicación: Sida 18(1): 101—103, f. 18. 1998.

Colección tipo: D.N. Smith 3808

Herbarios: MO, US; USM!

Nombre común: Desconocido.

Registro departamental: PA.

Regiones Ecológicas: BHA; 325 m.

SINANPE: Sin registro.

Herbarios peruanos: USM (isotipo).

Observaciones: Árbol conocido, al parecer, sólo de la colección tipo, una planta recolectada en la cuenca del Iscozacín, un tributario del Palcazú, muy cerca al límite oriental del Parque Nacional Yanachaga-Chemillén. Pipoly (1998) comentó sobre lo difícil de hacer una evaluación de conservación para esta especie, con lo que concordamos.

\section{Cybianthus tayoensis Pipoly \& Ricketson}

\section{CR, B2aD}

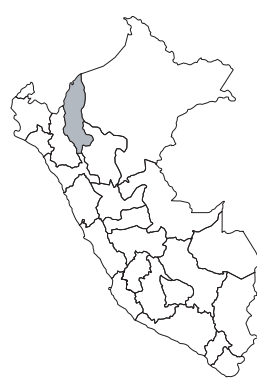

Publicación: Novon 16(2): 248-251, f. 1. 2006.

Colección tipo: H. van der Werff et al. 16229

Herbarios: FTG, MO; USM.

Nombre común: Desconocido.

Registro departamental: AM.

Regiones Ecológicas: BMHP; $800 \mathrm{~m}$.

SINANPE: Sin registro.

Herbarios peruanos: USM (isotipo citado).

Observaciones: Arbusto conocido de una sola población en la cima de una cordillera con suelos calcáreos. Pipoly \& Ricketson (2006) consideraron que esta especie debería considerarse en la categoría de En Peligro Crítico, por el área limitada de su distribución y el escaso número de individuos en la población. Aquí se concuerda con esa evaluación.

\section{Cybianthus vasquezii Pipoly}

\section{DD}

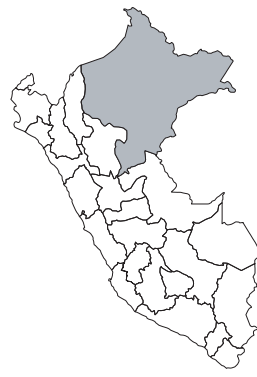

Publicación: Sida 18(1): 103-106, f. 19. 1998.

Colección tipo: R. Vásquez \& N. Jaramillo 1993

Herbarios: BRIT, F, MO, NY, US; AMAZ, USM.

Nombre común: Sésa, kurup.

Registro departamental: LO.

Regiones Ecológicas: BHA; $220 \mathrm{~m}$.

SINANPE: Sin registro.

Herbarios peruanos: AMAZ (isotipo citado), USM (isotipo citado).

Observaciones: Árbol dioico, conocido de dos localidades dispersas, en las cuencas del Pastaza y Corrientes. Pipoly (1998) menciona la carencia de información sobre las preferencias ecológicas de esta especie y dado el uso como tonificante entre los Mayna Jívaro, asume que es localmente común.

\section{Geissanthus spectabilis Pipoly}

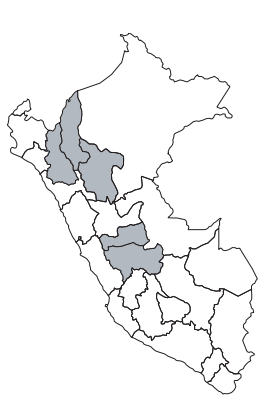

NT

Publicación: Sida 17(2): 463—465, f. 2. 1996. Colección tipo: B. Stein \& C. Todzia 2336 Herbarios: MO; USM.

Nombre común: Desconocido.

Registro departamental: AM, CA, JU, PA, SM.

Regiones Ecológicas: BMHM; 2050$2950 \mathrm{~m}$.

SINANPE: PNRA, STN

Herbarios peruanos: USM (isotipo citado+5).

Observaciones: Árbol conocido de varias localidades en el norte y centro del Perú, de las cuencas del Huallaga, Tulumayo, Palcazú y Marañón. Esta especie forma parte de la flora de dos áreas protegidas y probablemente cuente con poblaciones en el Parque Nacional Yanachaga-Chemillén.

\section{Myrsine brevis (J.F. Macbr.) Pipoly}

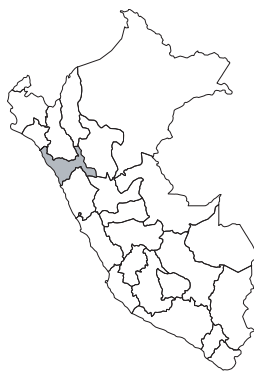

\section{CR, B1ab(iii)}

Publicación: Novon 2(4): 405. 1992.

Colección tipo: A. Weberbauer 7025

Herbarios: F; MOL!

Nombre común: Desconocido.

Registro departamental: LL.

Regiones Ecológicas: BPM; 3900-3700 m.

SINANPE: Sin registro.

Herbarios peruanos: MOL (isotipo).

Observaciones: Árbol o arbusto conocido, al parecer, sólo de la colección tipo, una planta recolectada en 1914, al sur del área que ocupa el Parque Nacional del Río Abiseo. Si bien debe aclararse sus vínculos con Myrsine andina y $M$. microdonta, se la reconoce aquí como un endemismo. Esta especie fue recolectada en fragmentos de bosque montano, los que están amenazados por incendios intencionales y tala.

\section{Myrsine bullata Pipoly}

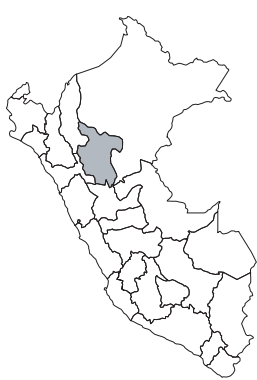

\section{EN, B1a}

Publicación: Novon 2(4): 399. 1992.

Colección tipo: K.R. Young 1552

Herbarios: F, MO; USM.

Nombre común: Desconocido.

Registro departamental: SM.

Regiones Ecológicas: BMHM; 3300$3400 \mathrm{~m}$.

SINANPE: PNRA

Herbarios peruanos: USM (isotipo citado).

Observaciones: Árbol conocido sólo de la localidad original, en la cuenca del Montecristo, en el Parque Nacional Río Abiseo. Ha sido recolectada en repetidas oportunidades de diferentes fragmentos de bosque, que si bien se hallan en un área protegida, no les exime de la amenaza de los incendios intencionales.

\section{Myrsine diazii Pipoly}

\section{EN, B1a}

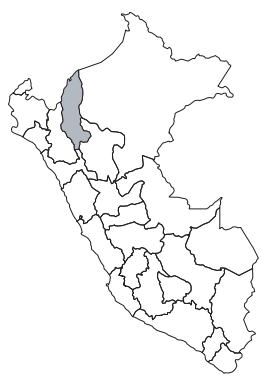

Publicación: Novon 2(4): 397-399, f. 4. 1992.

Colección tipo: C. Díaz \& J. Campos 3681 Herbarios: BISH, F, MO, US; AMAZ, USM!. Nombre común: Desconocido.

Registro departamental: AM.

Regiones Ecológicas: BMHM; 1900$2400 \mathrm{~m}$.

SINANPE: Sin registro.

Herbarios peruanos: AMAZ (isotipo citado+1), USM (isotipo). 
Observaciones: Árbol conocido de unas pocas localidades en la cuenca del Utcubamba, al parecer, asociado a los fragmentos de bosque montano. La deforestación podría ser una amenaza a sus poblaciones.

\section{Myrsine dilloniana Pipoly}

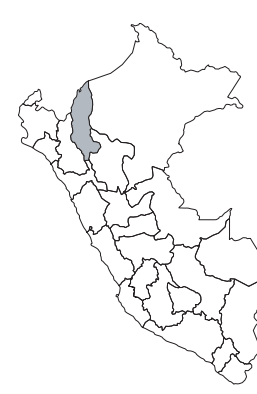

\section{CR, B1ab(iii)}

Publicación: Novon 2(4): 394, 396-397, f. 3. 1992.

Colección tipo: P.J. Barbour 3257

Herbarios: F, MO, TEX-LL; AMAZ, USM.

Nombre común: Desconocido.

Registro departamental: AM.

Regiones Ecológicas: BPM; $3200 \mathrm{~m}$.

SINANPE: ZRCC

Herbarios peruanos: AMAZ (isotipo citado), USM (isotipo citado).

Observaciones: Árbol o arbusto, conocido sólo de una planta recolectada en 1978, de una localidad, hoy ubicada, en la Zona Reservada Cordillera Colán. Esta es una zona con bosques montanos que reciben fuerte deforestación.

\section{Myrsine fosteri Pipoly}

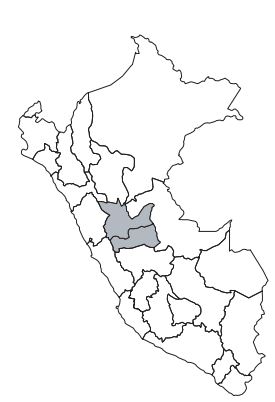

\section{EN, B1ab(iii)}

Publicación: Novon 2(4): 394. 1992.

Colección tipo: R.B. Foster et al. 7636

Herbarios: F, MO, NY; AMAZ, USM!.

Nombre común: Desconocido.

Registro departamental: HU, PA.

Regiones Ecológicas: BPM, BMHM; 2500-3000 m.

SINANPE: Sin registro.

Herbarios peruanos: AMAZ (isotipo citado), USM (isotipo).

Observaciones: Arbusto o árbol bajo conocido solamente de dos localidades, en las cuencas del Huallaga y del Paucartambo. No ha vuelto a ser recolectada desde 1983. La localidad original, al oeste de la ciudad de Oxapampa, cuenta con bosques fragmentados dada la actividad maderera.

\section{Myrsine microdonta Pipoly}

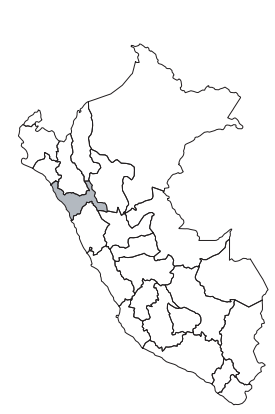

NE

Publicación: Novon 2(4): 392. 1992.

Colección tipo: A. Sagástegui A. \& M. Diestra 11903

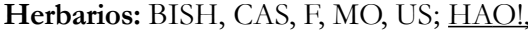
HUT.

Nombre común: Desconocido.

Registro departamental: LL.

Regiones Ecológicas: MA; $2800 \mathrm{~m}$.

SINANPE: Sin registro.

Herbarios peruanos: HUT (isotipo citado), HAO (isotipo).

Observaciones: Árbol o arbusto conocido sólo de la cuenca del Tablachaca. Esta especie tiene afinidades muy próximas, y probablemente sea coespecífica, con Myrsine brevis.

\section{Myrsine reynelii Pipoly \\ EN, B1ab(iii)}

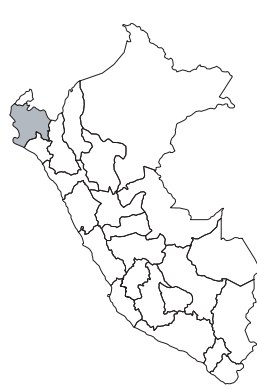

Publicación: Novon 2(4): 399, f. 5. 1992.

Colección tipo: C. Díaz 3180

Herbarios: MO; USM!.

Nombre común: Desconocido.

Registro departamental: PI.

Regiones Ecológicas: MA; 2000—2760

$\mathrm{m}$.

SINANPE: Sin registro.

Herbarios peruanos: HAO (1), USM (isotipo).

Observaciones: Árbol conocido de poblaciones aisladas y escasas, en las cuencas del Quiróz y Huancabamba, cerca al límite con Ecuador. Esta especie habita bosques montanos húmedos los que están siendo afectados por actividades mineras y agrícolas.

\section{Myrsine rivularis (Mez) Pipoly}

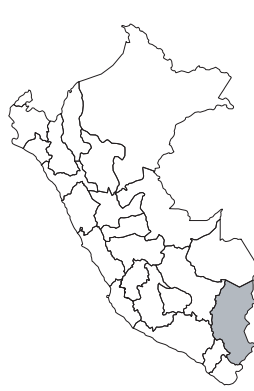

\section{DD}

Publicación: Novon 2(4): 406. 1992. Colección tipo: A. Weberbauer 1061

Herbarios: B.

Nombre común: Desconocido.

Registro departamental: PU.

Regiones Ecológicas: BMHM; altitud desconocida.

SINANPE: Sin registro.

Herbarios peruanos: Ninguno.

Observaciones: Arbusto conocido aparentemente sólo de la localidad original en la cuenca de un tributario del Inambari. El ejemplar original fue recolectado en 1902.

\section{Myrsine sessiliflora (Mez) Pipoly}

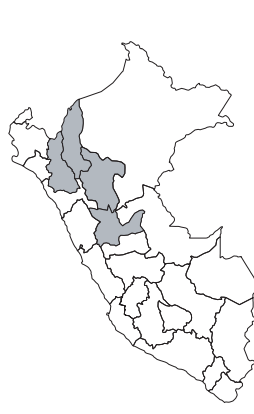

\section{NT}

Publicación: Novon 2(4): 406. 1992.

Colección tipo: A. Weberbauer 4324

Herbarios: B; MOL!.

Nombre común: Desconocido.

Registro departamental: AM, CA, HU, SM.

Regiones Ecológicas: MA, BMHM; 2500-3500 m.

SINANPE: Sin registro.

Herbarios peruanos: HUT (1), MOL (isotipo), MOLF (1).

Observaciones: Árbol conocido de varias localidades dispersas. Esta especie fue descrita de ejemplares recolectados en el norte del país, uno de ellos cerca al sitio arqueológico de Kuelap. Algunas poblaciones ocupan también los remanentes de bosques en la vertiente occidental, los que están fuertemente afectados por tala y quema.

\section{Myrsine weberbaueri (Mez) Pipoly}

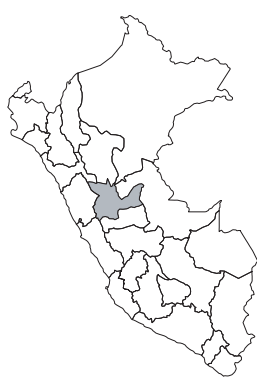

EN, B1ab(iii)

Publicación: Novon 2(4): 406. 1992.

Colección tipo: A. Weberbauer 3457

Herbarios: B; MOL!.

Nombre común: Desconocido.

Registro departamental: HU.

Regiones Ecológicas: BMHP; 800—1500 m.

SINANPE: Sin registro.

Herbarios peruanos: MOL (isotipo). 
Observaciones: Arbusto dioico, conocido sólo de dos localidades, en las cuencas del Monzón y Huallaga. Esta especie fue descrita de una planta recolectada en 1903, cerca a Monzón; esa cuenca no ha recibido suficiente estudio botánico, a pesar de albergar numerosos endemismos. Ambas localidades conocidas están afectadas por la deforestación asociada a la expansión agrícola.

\section{Myrsine youngii Pipoly}

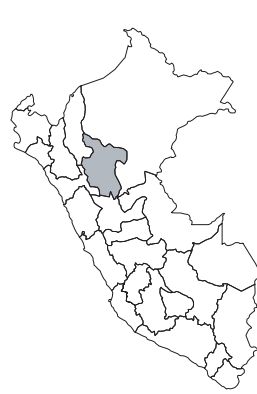

\section{NE}

Publicación: Novon 2(4): 402. 1992.

Colección tipo: K.R. Young \& B. León 4495

Herbarios: F, US; HUT!

Nombre común: Desconocido.

Registro departamental: SM.

Regiones Ecológicas: BMHM; 3100$3300 \mathrm{~m}$.

SINANPE: PNRA

Herbarios peruanos: HUT (isotipo).

Observaciones: Árbol conocido de una localidad en la vertiente oriental en el norte del país. La taxonomía de esta especie es dudosa.

\section{Stylogyne aguarunana Pipoly \& Ricketson}

\section{DD}

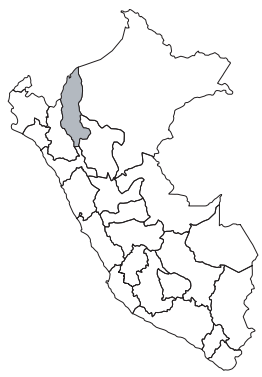

Publicación: Sida 19(2): 269-272, f.12. 2000.

Colección tipo: N. Jaramillo et al. 296

Herbarios: MO; CPUN.

Nombre común: Desconocido.

Registro departamental: AM.

Regiones Ecológicas: BHA; 320—480 m. SINANPE: Sin registro.

Herbarios peruanos: CPUN (isotipo+3), HUT (1).

Observaciones: Árbol conocido solamente de la cuenca del Marañón, donde es localmente rara. Parece habitar bosques con suelos de arena, por lo que se asume sus poblaciones sean fragmentadas, pero se desconoce la extensión de su rango. 